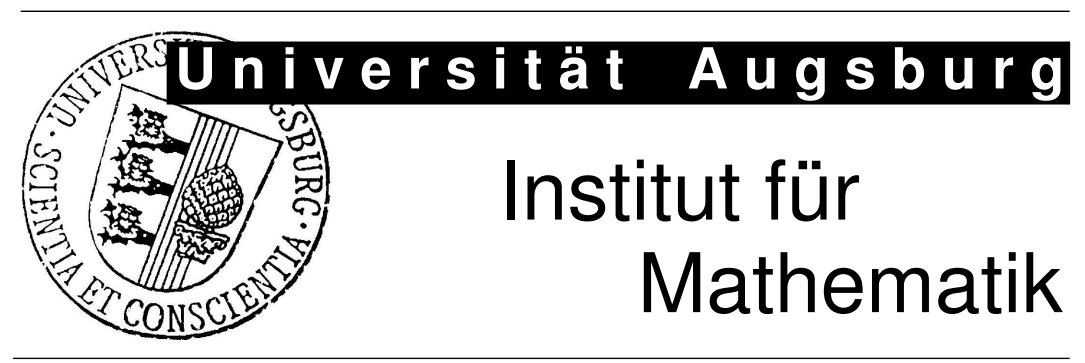

Ali Ünlü

A Note on Monotone Likelihood Ratio of the Total Score Variable in Unidimensional Item Response Theory 


\section{Impressum:}

Herausgeber:

Institut für Mathematik

Universität Augsburg

86135 Augsburg

http://www . math. uni-augsburg.de/forschung/preprint/

ViSdP:

Ali Ünlü

Institut für Mathematik

Universität Augsburg

86135 Augsburg

Preprint: Sämtliche Rechte verbleiben den Autoren (C) 2007 


\title{
A NOTE ON MONOTONE LIKELIHOOD
}

\author{
RATIO OF THE TOTAL SCORE \\ VARIABLE IN UNIDIMENSIONAL ITEM \\ RESPONSE THEORY
}

\begin{abstract}
This note provides a direct, elementary proof of the fundamental result on monotone likelihood ratio of the total score variable in unidimensional item response theory (IRT). This result is very important for practical measurement in IRT, because it justifies the use of the total score variable to order participants on the latent trait. The proof relies on a basic inequality for elementary symmetric functions which is proved by means of few purely algebraic, straightforward transformations. In particular, flaws in a proof of this result by Huynh [(1994). A new proof for monotone likelihood ratio for the sum of independent Bernoulli random variables. Psychometrika, 59, 77-79] are pointed out and corrected, and a natural generalization of the fundamental result to nonlinear (quasi-ordered) latent trait spaces is presented. This may be useful for multidimensional IRT or knowledge space theory, in which the latent 'ability' spaces are partially ordered with respect to, for instance, coordinate-wise vector-ordering or set-inclusion, respectively.
\end{abstract}




\section{Introduction}

Monotone likelihood ratio for the total score variable and latent trait plays an important role in item response theory (IRT). It implies stochastic ordering properties that can be conveniently interpreted in an IRT context (e.g., Hemker, Sijtsma, Molenaar, \& Junker, 1996, 1997; Hemker, Van der Ark, \& Sijtsma, 2001; Sijtsma, 1998; Van der Ark, 2001, 2005): Stochastic ordering of the total score variable by the latent trait, and stochastic ordering of the latent trait by the total score variable. The fundamental result (Ghurye \& Wallace, 1959; Grayson, 1988; Huynh, 1994) states that under mild conditions the total score variable has monotone likelihood ratio in the latent trait (Section 2). The (most recent and simplified) proof of this result by Huynh (1994), however, is flawed. In this note, a direct, elementary proof of the fundamental result is given (Section 3). It is different from the proof by Huynh (1994) in that it corrects flaws in Huynh's (1994) argument (Section 4) and easily allows for a generalization of the fundamental result to abstract nonlinear latent trait spaces (Section 5). ${ }^{1}$

Next, the required notation and terminology are introduced, and the monotone likelihood ratio and stochastic ordering properties are briefly reviewed. Throughout this note, only dichotomous items are considered. Let $X_{l}$ with realization $x_{l} \in\{0,1\}$ denote the item score variable for an item $I_{l}(1 \leq l \leq m$, $\left.m \in \mathbb{N}_{\geq 2}\right)$, and let $X_{+}:=\sum_{l=1}^{m} X_{l}$ with realization $x_{+} \in\{0,1, \ldots, m\}$ be the total score variable. A function $f:\{0,1, \ldots, m\} \rightarrow \mathbb{R}$ is nondecreasing if and

$\overline{1}$ Throughout this note, using the phrase 'nonlinear latent trait space' I refer to the definition of a nonlinear latent trait space given in Section 5 . 
only if (iff)

$$
\forall x, y \in\{0,1, \ldots, m\}, x \leq y: f(x) \leq f(y)
$$

Let the latent trait be denoted by $\theta, \theta \in \Theta \subseteq \mathbb{R}$. I refer to this as the assumption of unidimensionality. A function $f: \Theta \rightarrow \mathbb{R}$ is nondecreasing iff

$$
\forall \theta_{1}, \theta_{2} \in \Theta, \theta_{1} \leq \theta_{2}: f\left(\theta_{1}\right) \leq f\left(\theta_{2}\right)
$$

Let the conditional positive response probability $P\left(X_{l}=1 \mid \theta\right)$ as a function of $\theta \in \Theta$ be the item response function (IRF) of an item $I_{l}(1 \leq l \leq m)$. The assumption of local independence states that

$$
P\left(X_{1}=x_{1}, X_{2}=x_{2}, \ldots, X_{m}=x_{m} \mid \theta\right)=\prod_{l=1}^{m} P\left(X_{l}=x_{l} \mid \theta\right)
$$

for any $x_{l} \in\{0,1\}(1 \leq l \leq m)$ and $\theta \in \Theta$. The assumption of monotonicity holds iff any IRF $P\left(X_{l}=1 \mid.\right)(1 \leq l \leq m)$ is nondecreasing. ${ }^{2}$ Mokken's (1971) model of monotone homogeneity is based on the assumptions of unidimensionality, local independence, and monotonicity.

The total score variable $X_{+}$has monotone likelihood ratio (MLR) in $\theta$ iff, for

$\overline{2 \text { Here }}$ ' $P\left(X_{l}=1 \mid.\right)(1 \leq l \leq m)$ ' denotes the IRF

$$
P\left(X_{l}=1 \mid .\right): \Theta \rightarrow[0,1], \theta \mapsto P\left(X_{l}=1 \mid \theta\right)
$$

of an item $I_{l}(1 \leq l \leq m)$. 
any $0 \leq x_{+, 1} \leq x_{+, 2} \leq m,^{3}$

$$
\frac{P\left(X_{+}=x_{+, 2} \mid \theta\right)}{P\left(X_{+}=x_{+, 1} \mid \theta\right)}
$$

is a nondecreasing function of $\theta \in \Theta$. Similarly, the latent trait $\theta$ has $M L R$ in $X_{+}$iff, for any $\theta_{1}, \theta_{2} \in \Theta, \theta_{1} \leq \theta_{2}$,

$$
\frac{P\left(\theta_{2} \mid X_{+}=x_{+}\right)}{P\left(\theta_{1} \mid X_{+}=x_{+}\right)}
$$

is a nondecreasing function of $0 \leq x_{+} \leq m$. Because MLR of $X_{+}$in $\theta$ is equivalent to MLR of $\theta$ in $X_{+}$(Bayes' theorem), with abuse of terminology I simply speak of the 'property of MLR.' The fundamental result states that under mild conditions the total score variable has MLR in the latent trait (Theorem 1).

The MLR property is rather technical. Its importance in IRT actually stems $\overline{3}$ Throughout this note, mathematical expressions are assumed to be defined whenever they are written in the text. Note also that expressions such as $P\left(X_{+}=x_{+} \mid \theta\right)$ or $P\left(\theta \mid X_{+}=x_{+}\right)$(for $0 \leq x_{+} \leq m$ and $\theta \in \Theta$ ) are technically defined as follows. Let $f_{X_{+}, \Theta}\left(x_{+}, \theta\right)$ as a function of $0 \leq x_{+} \leq m$ and $\theta \in \Theta$ be the joint 'density function' of the random vector $\left(X_{+}, \Theta\right)$. Associated with this, define the marginal and conditional 'density functions' as follows:

$$
\begin{aligned}
f_{X_{+}}\left(x_{+}\right) & :=\int_{\theta \in \Theta} f_{X_{+}, \Theta}\left(x_{+}, \theta\right) d \theta \quad\left(0 \leq x_{+} \leq m\right), \\
f_{\Theta}(\theta) & :=\sum_{x_{+}=0}^{m} f_{X_{+}, \Theta}\left(x_{+}, \theta\right) \quad(\theta \in \Theta), \\
f_{X_{+} \mid \Theta}\left(x_{+} \mid \theta\right) & :=\frac{f_{X_{+}, \Theta}\left(x_{+}, \theta\right)}{f_{\Theta}(\theta)}\left(0 \leq x_{+} \leq m ; \text { for } \theta \in \Theta \text { with } f_{\Theta}(\theta) \neq 0\right), \\
f_{\Theta \mid X_{+}}\left(\theta \mid x_{+}\right) & :=\frac{f_{X_{+}, \Theta}\left(x_{+}, \theta\right)}{f_{X_{+}}\left(x_{+}\right)}\left(\theta \in \Theta ; \text { for } 0 \leq x_{+} \leq m \text { with } f_{X_{+}}\left(x_{+}\right) \neq 0\right) .
\end{aligned}
$$


from the fact that it implies the following two stochastic ordering properties that are much easier to interpret in IRT (cf. Section 5). The property of MLR implies that $X_{+}$is stochastically ordered by $\theta$. The stochastic ordering of the manifest variable $X_{+}$by $\theta(\mathrm{SOM})$ means that, for any $0 \leq x_{+} \leq m$,

$$
P\left(X_{+} \geq x_{+} \mid \theta\right)
$$

is a nondecreasing function of $\theta \in \Theta$. Note that this property takes the latent trait as a starting point. In practice, however, the total score variable is observed and inferences about the latent trait are required. The property of MLR also implies that $\theta$ is stochastically ordered by $X_{+}$. The stochastic ordering of the latent trait $\theta$ by $X_{+}$(SOL) means that, for any $\theta_{0} \in \Theta$,

$$
P\left(\theta \geq \theta_{0} \mid X_{+}=x_{+}\right)
$$

is a nondecreasing function of $0 \leq x_{+} \leq m$. Note that the property of SOL is very important for practical measurement, because it justifies the use of the total score variable to estimate the ordering of participants on the latent trait. In particular, this is the key result that justifies the use of Mokken's (1971) monotone homogeneity model as a measurement model for people. (The monotone homogeneity model satisfies the property of MLR and hence implies the SOL property.)

\section{Fundamental result}

The fundamental result on monotone likelihood ratio of the total score variable in unidimensional IRT (for dichotomous items) is as follows. 
Theorem 1 (Fundamental result) Under the assumptions of unidimensionality, local independence, and monotonicity, and the requirement that each item response function assumes values strictly between zero and one, the total score variable has monotone likelihood ratio in the (unidimensional) latent trait.

Proof. See Section 3.

Before giving a proof of this result (Section 3), some remarks with respect to Theorem 1 are in order.

1. The requirement of having IRFs assuming values strictly between zero and one is not that restrictive in practice. 'Boundary value' IRFs (assuming the values 0 and/or 1) may be closely approximated by IRFs that do meet this requirement.

2. Mokken's (1971) nonparametric monotone homogeneity model and hence parametric special cases such as the Rasch (1960) and Birnbaum (1968) models possess the MLR property.

3. Ghurye and Wallace (1959) first established the fundamental result. Their work stemmed from a problem posed by J. Loevinger on 'stochastic ordering of the latent trait' (for details, see Ghurye \& Wallace, 1959), and the fundamental result was obtained as a corollary of general results concerning a convolutive class of MLR families. Grayson (1988) provided a direct proof of a special case of Theorem 1, further adding the assumption that the first derivative of any IRF exists everywhere on the latent trait and is positive at some point of the latent trait. Grayson's (1988) proof is not only a proof of a special case of the fundamental result, but is also rather long and intricate. In particular, the added differentiability assumption excludes 
step-function IRFs as they arise from latent class measurement models such as the Lazarsfeld-Henry latent distance model (e.g., Heinen, 1996; Lazarsfeld \& Henry, 1968). Huynh (1994) provided a proof of the fundamental result which does not require the extra assumption made by Grayson (1988). Unfortunately, Huynh's (1994) proof is flawed (Section 4).

\section{Proof}

In this section, a simple proof of the fundamental result is presented.

Let $n \in \mathbb{N}_{\geq 1}$. Denote by $S_{r}(x)(0 \leq r \leq n)$ the $r$ th elementary symmetric function of $x=\left(x_{1}, x_{2}, \ldots, x_{n}\right) \in \mathbb{R}^{n}$; that is,

$$
\begin{aligned}
S_{0}(x) & :=1, \\
S_{1}(x) & :=\sum_{i=1}^{n} x_{i}, \\
S_{2}(x) & :=\sum_{1 \leq i<j \leq n} x_{i} x_{j}, \\
S_{3}(x) & :=\sum_{1 \leq i<j<k \leq n} x_{i} x_{j} x_{k}, \\
& \vdots \\
S_{n}(x) & :=\prod_{i=1}^{n} x_{i} .
\end{aligned}
$$

The proof of the fundamental result relies on the following basic inequality for elementary symmetric functions of which I give a simple proof. The proof is direct and elementary based on few purely algebraic, straightforward manipulations. ${ }^{4}$

$\overline{4}$ This inequality was originally suggested but justified in the wrong way by Huynh (1994); see Section 4. 
Lemma 1 (Basic inequality) If $1 \leq r \leq n$, then

$$
\frac{S_{r}(u+v)}{S_{r-1}(u+v)} \geq \frac{S_{r}(u)}{S_{r-1}(u)}
$$

for any $u=\left(u_{1}, u_{2}, \ldots, u_{n}\right) \in \mathbb{R}_{\geq 0}^{n}$ for which $S_{r-1}(u) \neq 0$ (e.g., for positive $u \in \mathbb{R}_{>0}^{n}$, as in the proof of Theorem 1$)$, and for any $v=\left(v_{1}, v_{2}, \ldots, v_{n}\right) \in \mathbb{R}_{\geq 0}^{n}$.

Proof. Without restriction, let $2 \leq r \leq n$. I have to show that

$$
\frac{\sum_{1 \leq i_{1}<\ldots<i_{r} \leq n} \prod_{j=1}^{r}\left(u_{i_{j}}+v_{i_{j}}\right)}{\sum_{1 \leq i_{1}^{\prime}<\ldots<i_{r-1}^{\prime} \leq n} \prod_{j^{\prime}=1}^{r-1}\left(u_{i_{j^{\prime}}^{\prime}}+v_{i_{j^{\prime}}^{\prime}}\right)} \geq \frac{\sum_{1 \leq i_{1}^{\prime \prime}<\ldots<i_{r}^{\prime \prime} \leq n} \prod_{j^{\prime \prime}=1}^{r} u_{i_{j^{\prime \prime}}^{\prime \prime}}}{\sum_{1 \leq i_{1}^{\prime \prime \prime}<\ldots<i_{r-1}^{\prime \prime \prime} \leq n} \prod_{j^{\prime \prime \prime}=1}^{r-1} u_{i_{j^{\prime \prime \prime}}^{\prime \prime \prime}}} .
$$

This inequality is equivalent to

$$
\begin{gathered}
\sum_{1 \leq i_{1}^{\prime \prime \prime}<\ldots<i_{r-1}^{\prime \prime \prime} \leq n} \sum_{1 \leq i_{1}<\ldots<i_{r} \leq n}\left(\prod_{j^{\prime \prime \prime}=1}^{r-1} u_{i_{j^{\prime \prime \prime}}^{\prime \prime \prime}}\right)\left(\prod_{j=1}^{r}\left(u_{i_{j}}+v_{i_{j}}\right)\right) \\
\geq \sum_{1 \leq i_{1}^{\prime}<\ldots<i_{r-1}^{\prime} \leq n} \sum_{1 \leq i_{1}^{\prime \prime}<\ldots<i_{r}^{\prime \prime} \leq n}\left(\prod_{j^{\prime}=1}^{r-1}\left(u_{i_{j^{\prime}}^{\prime}}+v_{i_{j^{\prime}}}\right)\right)\left(\prod_{j^{\prime \prime}=1}^{r} u_{i_{j^{\prime \prime}}^{\prime \prime}}\right) .
\end{gathered}
$$

For any choices of $1 \leq i_{1}^{\prime}<\ldots<i_{r-1}^{\prime} \leq n$ and $1 \leq i_{1}^{\prime \prime}<\ldots<i_{r}^{\prime \prime} \leq n$, there is an appropriate $i_{\hat{j}^{\prime \prime}}^{\prime \prime}\left(1 \leq \widehat{j^{\prime \prime}} \leq r\right)$ such that $i_{j^{\prime \prime}}^{\prime \prime} \neq i_{j^{\prime}}^{\prime}$ for any $1 \leq j^{\prime} \leq r-1$. For such a list of indices, for the right-hand side of the previous inequality I finally have

$$
\begin{aligned}
& \sum_{1 \leq i_{1}^{\prime}<\ldots<i_{r-1}^{\prime} \leq n} \sum_{1 \leq i_{1}^{\prime \prime}<\ldots<i_{r}^{\prime \prime} \leq n}\left(\prod_{j^{\prime}=1}^{r-1}\left(u_{i_{j^{\prime}}^{\prime}}+v_{i_{j^{\prime}}}\right)\right)\left(\prod_{j^{\prime \prime}=1}^{r} u_{i_{j^{\prime \prime}}^{\prime \prime}}\right) \\
\leq & \sum_{1 \leq i_{1}^{\prime}<\ldots<i_{r-1}^{\prime} \leq n} \sum_{1 \leq i_{1}^{\prime \prime}<\ldots<i_{r}^{\prime \prime} \leq n}\left(u_{i_{j^{\prime \prime}}^{\prime \prime}}+v_{i_{j^{\prime \prime}}^{\prime \prime}}\right)\left(\prod_{j^{\prime}=1}^{r-1}\left(u_{i_{j^{\prime}}^{\prime}}+v_{i_{j^{\prime}}^{\prime}}\right)\right)\left(\prod_{1 \leq j^{\prime \prime} \leq r, j^{\prime \prime} \neq \hat{j}^{\prime \prime}} u_{i_{j^{\prime \prime}}^{\prime \prime}}\right) \\
\leq & \sum_{1 \leq i_{1}^{\prime \prime \prime}<\ldots<i_{r-1}^{\prime \prime \prime} \leq n} \sum_{1 \leq i_{1}<\ldots<i_{r} \leq n}\left(\prod_{j^{\prime \prime \prime}=1}^{r-1} u_{i_{j^{\prime \prime \prime}}^{\prime \prime \prime}}\right)\left(\prod_{j=1}^{r}\left(u_{i_{j}}+v_{i_{j}}\right)\right) .
\end{aligned}
$$

The basic inequality is at the core of the following proof of Theorem 1 . 
Proof of Theorem 1. Let $\theta \in \Theta$ and $0 \leq x_{+} \leq m$. As described in Huynh (1994), the term $P\left(X_{+}=x_{+} \mid \theta\right)$ can be expressed as

$$
P\left(X_{+}=x_{+} \mid \theta\right)=f(\theta) \cdot \sum_{x \in \mathcal{R}_{x_{+}}} \prod_{l=1}^{m}\left[\xi_{l}(\theta)^{x_{l}}\right]=f(\theta) \cdot S_{x_{+}}(\xi(\theta))
$$

where

$$
\begin{aligned}
f(\theta) & :=\prod_{l=1}^{m}\left[1-P\left(X_{l}=1 \mid \theta\right)\right], \\
\mathcal{R}_{x_{+}} & :=\left\{x=\left(x_{1}, x_{2}, \ldots, x_{m}\right) \in\{0,1\}^{m}: \sum_{l=1}^{m} x_{l}=x_{+}\right\}, \\
\xi_{l}(\theta) & :=\frac{P\left(X_{l}=1 \mid \theta\right)}{1-P\left(X_{l}=1 \mid \theta\right)} \quad(1 \leq l \leq m),
\end{aligned}
$$

and $S_{x_{+}}(\xi(\theta))$ is the $x_{+}$th elementary symmetric function of the positive vector $\xi(\theta):=\left(\xi_{1}(\theta), \xi_{2}(\theta), \ldots, \xi_{m}(\theta)\right) \in \mathbb{R}_{>0}^{m}$. (At this point, the assumption of local independence and the requirement that IRFs assume values strictly between zero and one are applied.) Let $0 \leq x_{+, 1}<x_{+, 2} \leq m$, and let $\theta_{1}, \theta_{2} \in \Theta$ with $\theta_{1}<\theta_{2}$. By the assumption of monotonicity, $P\left(X_{l}=1 \mid \theta_{1}\right) \leq P\left(X_{l}=1 \mid \theta_{2}\right)$ for any $1 \leq l \leq m$. Hence, $\xi_{l}\left(\theta_{1}\right) \leq \xi_{l}\left(\theta_{2}\right)$ for any $1 \leq l \leq m$. In particular, $\xi_{l}\left(\theta_{2}\right)=\xi_{l}\left(\theta_{1}\right)+v_{l}$, for $v_{l} \in \mathbb{R}_{\geq 0}(1 \leq l \leq m)$. That is, $\xi\left(\theta_{2}\right)=\xi\left(\theta_{1}\right)+v$, for $v=\left(v_{1}, v_{2}, \ldots, v_{m}\right) \in \mathbb{R}_{\geq 0}^{m}$.

Based on Lemma 1, the property of MLR can now be proved:

$$
\begin{aligned}
& \frac{P\left(X_{+}=x_{+, 2} \mid \theta_{2}\right)}{P\left(X_{+}=x_{+, 1} \mid \theta_{2}\right)} \\
& \stackrel{\text { Eq. (1) }}{=} \frac{S_{x_{+, 2}}\left(\xi\left(\theta_{2}\right)\right)}{S_{x_{+, 1}}\left(\xi\left(\theta_{2}\right)\right)} \\
& \quad=\frac{S_{x_{+, 2}}\left(\xi\left(\theta_{1}\right)+v\right)}{S_{x_{+, 2}-1}\left(\xi\left(\theta_{1}\right)+v\right)} \cdot \frac{S_{x_{+, 2}-1}\left(\xi\left(\theta_{1}\right)+v\right)}{S_{x_{+, 2}-2}\left(\xi\left(\theta_{1}\right)+v\right)} \cdot \ldots \cdot \frac{S_{x_{+, 1}+1}\left(\xi\left(\theta_{1}\right)+v\right)}{S_{x_{+, 1}}\left(\xi\left(\theta_{1}\right)+v\right)} \\
& \quad \stackrel{\text { Lemma 1 }}{\geq} \frac{S_{x_{+, 2}}\left(\xi\left(\theta_{1}\right)\right)}{S_{x_{+, 2}-1}\left(\xi\left(\theta_{1}\right)\right)} \cdot \frac{S_{x_{+, 2}-1}\left(\xi\left(\theta_{1}\right)\right)}{S_{x_{+, 2}-2}\left(\xi\left(\theta_{1}\right)\right)} \cdot \ldots \cdot \frac{S_{x_{+, 1}+1}\left(\xi\left(\theta_{1}\right)\right)}{S_{x_{+, 1}}\left(\xi\left(\theta_{1}\right)\right)}
\end{aligned}
$$




$$
\begin{aligned}
& =\frac{S_{x_{+, 2}}\left(\xi\left(\theta_{1}\right)\right)}{S_{x_{+, 1}}\left(\xi\left(\theta_{1}\right)\right)} \\
& \stackrel{\text { Eq. (1) }}{=} \frac{P\left(X_{+}=x_{+, 2} \mid \theta_{1}\right)}{P\left(X_{+}=x_{+, 1} \mid \theta_{1}\right)} .
\end{aligned}
$$

Before contrasting this proof with Huynh's (1994) argument (Section 4), it is instructive to see what prerequisites in Theorem 1 are actually necessary to prove the theorem. Obviously, the proof rests on the assumptions of local independence and monotonicity, and the requirement that IRFs assume values strictly between zero and one. However, the algebraic (e.g., addition) and extra order-theoretic (e.g., completeness) properties underlying the assumption of unidimensionality are not required for the proof: ${ }^{5}$ The property of MLR is solely formulated 'locally,' that is, for 'pairs' of latent trait points $\theta_{1}, \theta_{2} \in \Theta$ which are in $\leq$-relation $\theta_{1}<\theta_{2}$. In fact, a variant of Theorem 1 also applies in a more general setting where the assumption of unidimensionality is weakened to allow for abstract nonlinear latent trait spaces (Section 5).

\section{Correction to Huynh's (1994) argument}

Huynh's (1994) proof of the fundamental result (Theorem 1) is wrongly based on the following inequality for elementary symmetric functions by Marcus and Lopes (1957).

$\overline{5}$ The assumption of unidimensionality is here understood as the mathematical structure $\left\langle\Theta \subseteq \mathbb{R},+,-, 0, \cdot{ }^{-1}, 1, \leq\right\rangle$. 
Theorem 2 (Marcus-Lopes inequality) If $1 \leq r \leq n$, then

$$
\frac{S_{r}(u+v)}{S_{r-1}(u+v)} \geq \frac{S_{r}(u)}{S_{r-1}(u)}+\frac{S_{r}(v)}{S_{r-1}(v)}
$$

for any $u=\left(u_{1}, u_{2}, \ldots, u_{n}\right)$ and $v=\left(v_{1}, v_{2}, \ldots, v_{n}\right)$ in $\mathbb{R}_{\geq 0}^{n}$, where for each of these vectors at least $\boldsymbol{r}$ of its elements are assumed to be positive.

Proof. See Marcus and Lopes (1957, pp. 306-307, Theorem 1).

In the context of the fundamental result, the Marcus-Lopes inequality is not applicable. Huynh's (1994) conclusion of the basic inequality (Lemma 1) from the Marcus-Lopes inequality is wrong and not justified. In Theorem 1, IRFs are assumed to be nondecreasing functions. Hence in the proof one can have $\xi\left(\theta_{2}\right)=\xi\left(\theta_{1}\right)+v\left(\right.$ for $\left.\theta_{1}, \theta_{2} \in \Theta, \theta_{1}<\theta_{2}\right)$, where $v=\left(v_{1}, v_{2}, \ldots, v_{m}\right) \in \mathbb{R}_{\geq 0}^{m}$ may, in principle, contain any number $1 \leq L \leq m$ of zeros. Theorem 2, however, requires at least $r$ of the elements $v_{l}(1 \leq l \leq m)$ to be positive.

An appreciation for this issue can be gained by the following example. Consider $m:=4$ dichotomous items. Let $x_{+, 1}:=2$ and $x_{+, 2}:=3$. Let $\theta_{1}, \theta_{2} \in \Theta, \theta_{1}<\theta_{2}$, with $P\left(X_{1}=1 \mid \theta_{1}\right)<P\left(X_{1}=1 \mid \theta_{2}\right)$ and $P\left(X_{l}=1 \mid \theta_{1}\right)=P\left(X_{l}=1 \mid \theta_{2}\right)$ for any $2 \leq l \leq 4$. Then, $\xi\left(\theta_{2}\right)=\xi\left(\theta_{1}\right)+v$, with $v=\left(v_{1}, 0,0,0\right)$ and $v_{1}>0$. Under these conditions,

$$
\frac{P\left(X_{+}=3 \mid \theta_{2}\right)}{P\left(X_{+}=2 \mid \theta_{2}\right)}=\frac{S_{3}\left(\xi\left(\theta_{2}\right)\right)}{S_{2}\left(\xi\left(\theta_{2}\right)\right)}=\frac{S_{3}\left(\xi\left(\theta_{1}\right)+v\right)}{S_{2}\left(\xi\left(\theta_{1}\right)+v\right)} .
$$

But the Marcus-Lopes inequality cannot be used to conclude that

$$
\frac{S_{3}\left(\xi\left(\theta_{1}\right)+v\right)}{S_{2}\left(\xi\left(\theta_{1}\right)+v\right)} \geq \frac{S_{3}\left(\xi\left(\theta_{1}\right)\right)}{S_{2}\left(\xi\left(\theta_{1}\right)\right)}+\frac{S_{3}(v)}{S_{2}(v)}
$$


in order to obtain the required statement

$$
\frac{P\left(X_{+}=3 \mid \theta_{2}\right)}{P\left(X_{+}=2 \mid \theta_{2}\right)} \geq \frac{S_{3}\left(\xi\left(\theta_{1}\right)\right)}{S_{2}\left(\xi\left(\theta_{1}\right)\right)}+\frac{S_{3}(v)}{S_{2}(v)} \geq \frac{S_{3}\left(\xi\left(\theta_{1}\right)\right)}{S_{2}\left(\xi\left(\theta_{1}\right)\right)}=\frac{P\left(X_{+}=3 \mid \theta_{1}\right)}{P\left(X_{+}=2 \mid \theta_{1}\right)}
$$

The reason for this fact is that only one element of $v$, namely $v_{1}$, is positive, contrary to at least three positive elements as required for the Marcus-Lopes inequality to hold. Note also that the summand $S_{3}(v) / S_{2}(v)$ on the right-hand side of the Marcus-Lopes inequality is not well-defined since the denominator $S_{2}(v)$ is equal to zero.

Furthermore, Huynh's (1994) reference to Marshall and Olkin (1979, p. 80) utilises the Marcus-Lopes inequality for more restrictive vectors all of the elements of which are assumed to be positive.

In sum, the basic inequality is the crucial statement solely required for the proof of the fundamental result. Though this inequality is elementary, it cannot be derived from the Marcus-Lopes inequality. The basic inequality has to be proved separately. In the note, this is accomplished by the simple proof of Lemma 1.

\section{Generalized fundamental result}

Finally, the fundamental result is extended to the case of nonlinear latent trait spaces.

A nonlinear latent trait space is a pair $(\Theta, \preceq)$, with $\Theta$ a nonempty set of latent trait points, and $\preceq$ a quasi-order (reflexive and transitive binary relation) 
on $\Theta$. I refer to this as the assumption of nonlinear dimensionality. ${ }^{6}$ The unidimensional concepts introduced in Section 1 can be formulated for abstract nonlinear latent trait spaces. For instance, a function $f: \Theta \rightarrow \mathbb{R}$ is isotonic iff

$$
\forall \theta_{1}, \theta_{2} \in \Theta, \theta_{1} \preceq \theta_{2}: f\left(\theta_{1}\right) \leq f\left(\theta_{2}\right) \cdot{ }^{7}
$$

Or, the assumption of isotonicity means that all IRFs are isotonic.

The generalized fundamental result (for dichotomous items) is as follows.

Corollary 1 (Generalized fundamental result) Under the assumptions of nonlinear dimensionality, local independence, and isotonicity, and the requirement that each item response function assumes values strictly between zero and one, the total score variable has monotone likelihood ratio in the (nonlinear) latent trait.

Proof. The proof of Theorem 1 carries over easily (cf. Section 3).

Discussion. What can be gained from such a generalization of the fundamental result?

1. The generalized fundamental result is at the interface of IRT and knowledge

$\overline{6}$ Important examples of nonlinear latent trait spaces are provided by Euclidean subsets $\Theta \subseteq \mathbb{R}^{n}\left(n \in \mathbb{N}_{\geq 2}\right)$ which are partially ordered with respect to, for instance, coordinate-wise vector-ordering (multidimensional IRT), or by set-families which are partially ordered with respect to set-inclusion (knowledge space theory). The class of knowledge space theory examples is discussed in more detail below.

7 For $\preceq$-incomparable latent trait points $\theta_{1}, \theta_{2} \in \Theta$, that is, $\theta_{1} \npreceq \theta_{2}$ and $\theta_{2} \npreceq \theta_{1}$, no restrictions are imposed on the relationship of the function values $f\left(\theta_{1}\right)$ and $f\left(\theta_{2}\right)$ to each other. 
space theory (KST), two modern but still split directions of psychological test theories. ${ }^{8}$ It provides a first connection between the two theories on a nonparametric probabilistic basis. The KST variant of the generalized fundamental result (for dichotomous items) takes the following form.

\section{Corollary 2 (KST variant of generalized fundamental result) Let $Q$} denote the item set $\left\{I_{l}: 1 \leq l \leq m\right\}$. Let $\mathcal{K}$ be a knowledge structure on $Q$, that is, a family of subsets of $Q$ containing at least the empty set $\emptyset$ and $Q$. Then, for the nonlinear latent trait space $(\mathcal{K}, \subseteq)$, under the assumptions of local independence and isotonicity, and the requirement that $0<P\left(X_{l}=1 \mid K\right)<1$ for any $K \in \mathcal{K}$ and $1 \leq l \leq m$, the total score variable has monotone likelihood ratio in the (nonlinear) latent trait $K \in \mathcal{K}$.

Proof. Here, $\Theta:=\mathcal{K}$ and $\preceq:=\subseteq$.

2. As mentioned in Section 1, the MLR property is rather technical and its (hence the fundamental result's) importance in IRT actually stems from the fact that it implies the SOM and SOL properties (unidimensional case). Whether the generalized MLR property still implies the generalized SOM and/or generalized SOL properties, that is, whether the generalized fundamental result may still apply, (nonlinear case) is thoroughly investigated in Ünlü (2006). Some of the relevant findings are (for details, see Ünlü, 2006): (a) The generalized fundamental result implies stochastic ordering of the total score variable by the nonlinear latent trait, but may fail to imply

$\overline{8 \text { KST }}$ was introduced by Doignon and Falmagne (1985); see also Doignon and Falmagne (1999) and Falmagne, Koppen, Villano, Doignon, and Johannesen (1990). 
stochastic ordering of the nonlinear latent trait by the total score variable.

(b) The reason for this fact (order-theoretic completeness property) and (technical) conditions under which the implication holds are specified. (c) Simulations demonstrate that violations of the generalized SOL property occur only for extreme (unrealistic) parameter vectors.

In any case, an extension of unidimensional ordering properties to the case of incomparabilities among latent trait points may prove valuable in nonlinear settings such as multidimensional IRT or KST.

\section{References}

Birnbaum, A. (1968). Some latent trait models and their use in inferring an examinee's ability. In F. M. Lord, \& M. R. Novick (Eds.), Statistical theories of mental test scores (pp. 397-479). Reading, MA: Addison-Wesley.

Doignon, J.-P., \& Falmagne, J.-Cl. (1985). Spaces for the assessment of knowledge. International Journal of Man-Machine Studies, 23, 175-196.

Doignon, J.-P., \& Falmagne, J.-Cl. (1999). Knowledge spaces. Berlin: Springer.

Falmagne, J.-Cl., Koppen, M., Villano, M., Doignon, J.-P., \& Johannesen, L. (1990). Introduction to knowledge spaces: How to build, test and search them. Psychological Review, 97, 201-224.

Ghurye, S. G., \& Wallace, D. L. (1959). A convolutive class of monotone likelihood ratio families. The Annals of Mathematical Statistics, 30, 11581164.

Grayson, D. A. (1988). Two-group classification in latent trait theory: Scores with monotone likelihood ratio. Psychometrika, 53, 383-392.

Heinen, T. (1996). Latent class and discrete latent trait models: Similarities 
and differences. Thousand Oaks: Sage Publications.

Hemker, B. T., Sijtsma, K., Molenaar, I. W., \& Junker, B. W. (1996). Polytomous IRT models and monotone likelihood ratio of the total score. Psychometrika, 61, 679-693.

Hemker, B. T., Sijtsma, K., Molenaar, I. W., \& Junker, B. W. (1997). Stochastic ordering using the latent trait and the sum score in polytomous IRT models. Psychometrika, 62, 331-347.

Hemker, B. T., Van der Ark, L. A., \& Sijtsma, K. (2001). On measurement properties of continuation ratio models. Psychometrika, 66, 487-506.

Huynh, H. (1994). A new proof for monotone likelihood ratio for the sum of independent Bernoulli random variables. Psychometrika, 59, 77-79.

Lazarsfeld, P. F., \& Henry, N. W. (1968). Latent structure analysis. Boston: Houghton Mifflin.

Marcus, M., \& Lopes, L. (1957). Inequalities for symmetric functions and Hermitian matrices. Canadian Journal of Mathematics, 9, 305-312.

Marshall, A. W., \& Olkin, I. (1979). Inequalities: Theory of majorization and its applications. New York: Academic Press.

Mokken, R. J. (1971). A theory and procedure of scale analysis. The Hague, Mouton/Berlin: De Gruyter.

Rasch, G. (1960). Probabilistic models for some intelligence and attainment tests. Copenhagen: Nielsen \& Lydiche.

Sijtsma, K. (1998). Methodology review: Nonparametric IRT approaches to the analysis of dichotomous item scores. Applied Psychological Measurement, 22, 3-31.

Ünlü, A. (2006). Nonparametric IRT axioms and properties under nonlinearity and their exemplification with knowledge space theory. Manuscript submitted for publication. 
Van der Ark, L. A. (2001). Relationships and properties of polytomous item response theory models. Applied Psychological Measurement, 25, 273-282. Van der Ark, L. A. (2005). Stochastic ordering of the latent trait by the sum score under various polytomous IRT models. Psychometrika, 70, 283-304. 